UCRL-JC-119372

PREPRINT

\title{
Dynamics of Gas-Filled Hohlraums
}

T. J. Orzechowski, R. L. Kauffman, R. K. Kirkwood, H. N. Kornblum, W. K. Levedahl, D. S. Montgomery, L. V. Powers, T. D. Shepard, G. F. Stone, L. J. Suter, R. J. Wallace, J. M. Foster, and P. Rosen

This paper was prepared for submittal to the LIRPP ‘95

Osaka, Japan

April 24-28, 1995

April 24, 1995

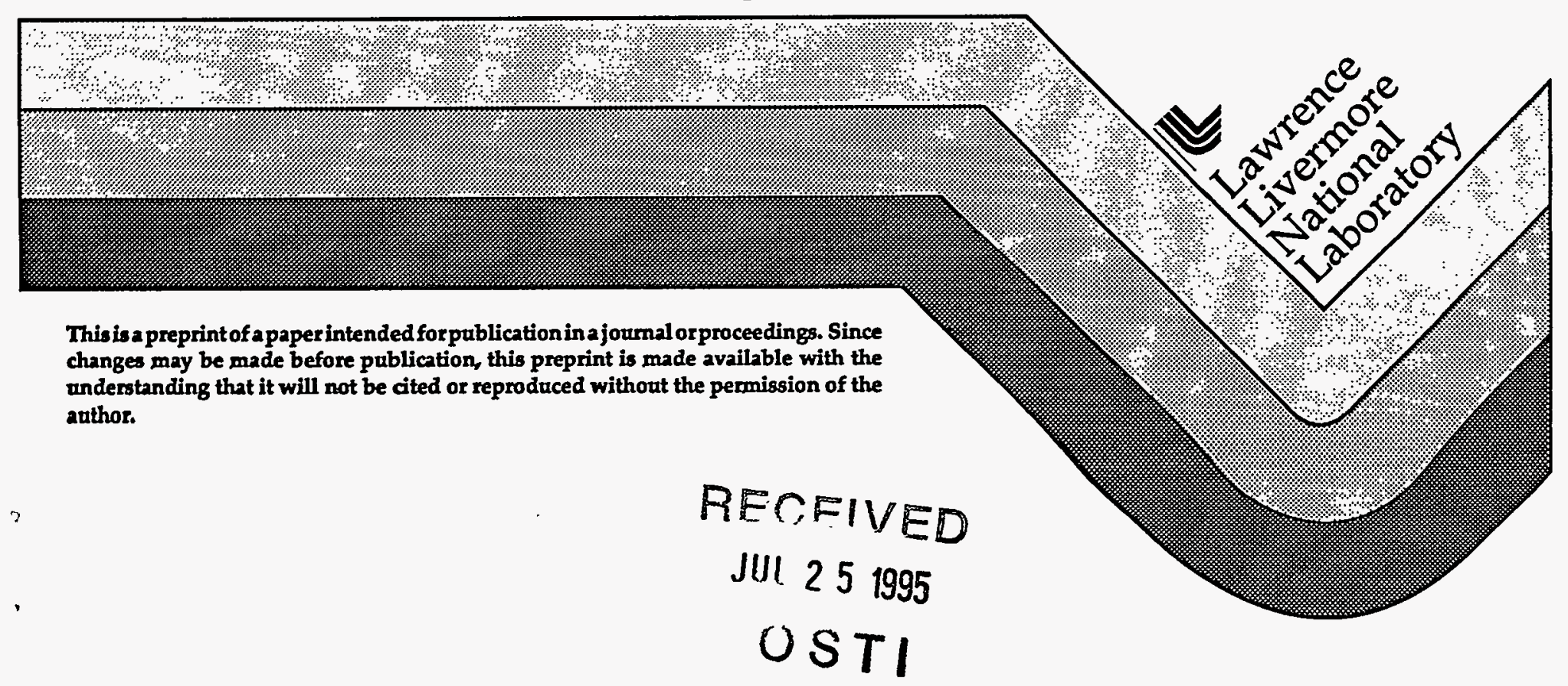




\section{DISCLAIMER}

This document was prepared as an account of work sponsored by an agency of the United States Government. Neither the United States Government nor the University of California nor any of their employees, makes any warranty, express or implied, or assumes any legal liability or responsibility for the accuracy, completeness, or usefulness of any information, apparatus, product, or process disclosed, or represents that its use would not infringe privately owned rights. Reference herein to any specific commercial product, process, or service by trade name, trademark, manufacturer, or otherwise, does not necessarily constitute or imply its endorsement, recommendation, or favoring by the United States Government or the University of California. The views and opinions of authors expressed herein do not necessarily state or reflect those of the United States Government or the University of California, and shall not be used for advertising or product endorsement purposes. 


\section{DISCLAIMER}

Portions of this document may be illegible in electronic image products. Images are produced from the best available original document. 


\title{
Dynamics of gas-filled hohlraums
}

\author{
T. J. Orzechowski, R. L. Kauffman, R. K. Kirkwood, H. N. \\ Kornblum, W. K. Levedahl, D. S. Montgomery, L. V. Powers, T. \\ D. Shepard, G. F. Stone, L. J. Suter, R. J. Wallace \\ Lawrence Livermore National Laboratory \\ P. O. Box 808 \\ Livermore, $C A 94550$
}

J. M. Foster, P. Rosen

Atomic Weapons Establishment

Aldermaston, $U . K$.

In order to prevent high- $Z$ plasma from filling in the hohlraum in indirect drive experiments, a low- $\mathrm{Z}$ material, or tamper is introduced into the hohlraum. This material, when fully ionized is typically less than one-tenth of the critical density for the laser light used to illuminate the hohlraum. This tamper absorbs little of the laser light, thus allowing most of the laser energy to be absorbed in the high- $Z$ material. However, the pressure associated with this tamper is sufficient to keep the hohlraum wall material from moving a significant distance into the interior of the hohlraum. In this paper we discuss measurements of the motion of the interface between the tamper and the high- $Z$ hohlraum material. We also present measurements of the effect the tamper has on the hohlraum temperature.

\section{INTRODUCTION}

In the indirect drive approach to inertial confinement fusion, the fuel pellet is imploded by $\mathrm{X}$-ray driven ablation inside a high-Z cavity, or hohlraum (1). This $\mathrm{X}-$ ray drive is generated by the interaction of intense laser beams with the hohlraum wall interior. In addition to ablating the fuel pellet surface, this radiation heats and ablates the hohlraum wall causing the hohlraum to fill with a dense, high-Z plasma. This plasma can refract the incident laser beams or cause the absorption region to move a significant distance from the hohlraum wall. Both of these situations can affect the drive symmetry. If the density gets too high, parametric instabilities can scatter the laser light, affecting both symmetry and energy balance.

To prevent the ablated hohlraum wall material from filling the cavity, a low$\mathrm{Z}$ material, or tamper, is used to hold back the high- $\mathrm{Z}$ hohlraum wall material. This tamper can be generated from a solid, such as $\mathrm{CH}$, coated on the interior hohlraum wall, or gas such as methane, confined in the hohlraum. These gas-filled hohlraums must be designed with windows to confine the gas. The tamper must allow the laser light to penetrate to the hohlraum wall, but must be of sufficient density and temperature to hold back the ablated high- $Z$ material. A gas filled hohlraum is shown schematically in figure 1 . Thin (3500 $\AA$ ) polyimide $\left(\mathrm{C}_{22} \mathrm{H}_{10} \mathrm{~N}_{2} \mathrm{O}_{5}\right)$ windows cover the laser entrance holes (LEHs) to provide a gastight seal. The laser light rapidly burns through the windows and gas, heating and ionizing the low- $\mathrm{Z}$ material as it propagates to the hohlraum wall. In the experiments discussed here, this occurs in about $200 \mathrm{ps}$. 


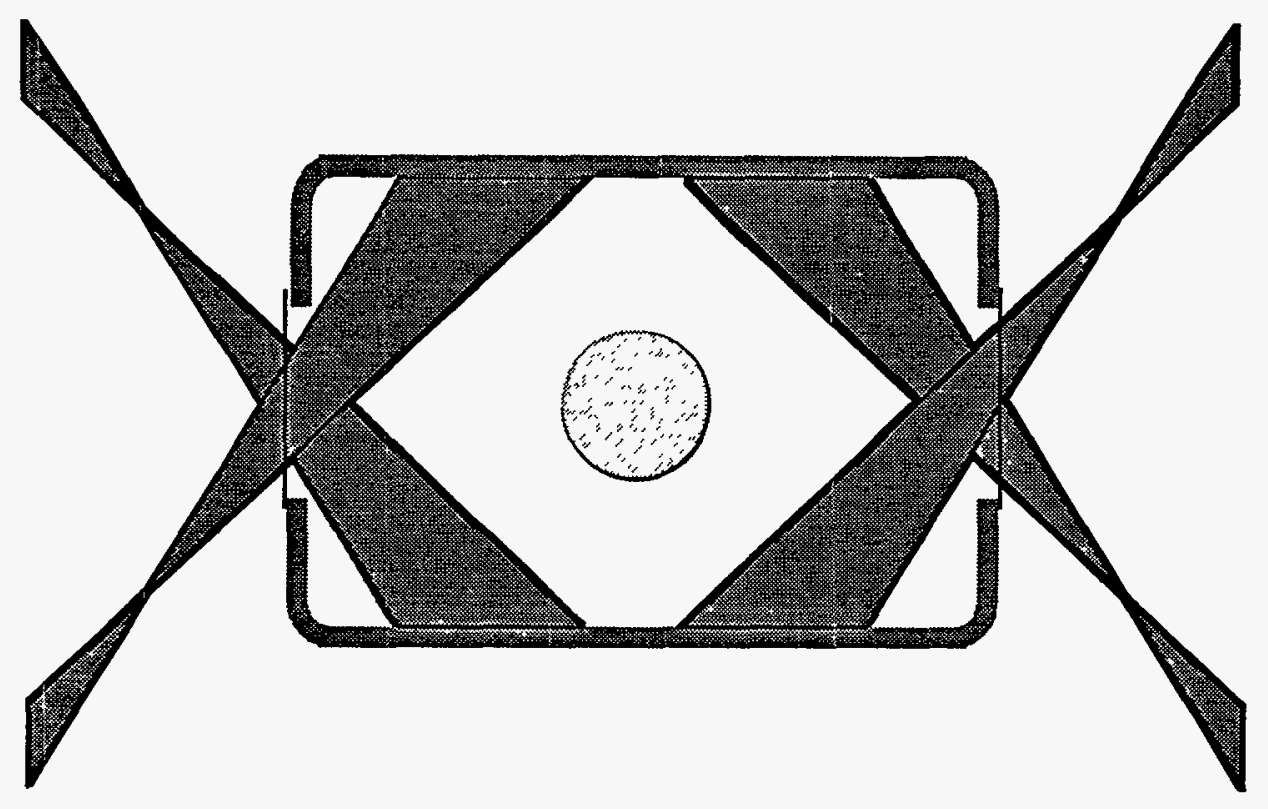

FIGURE 1. Gas-filled hohlraum. The laser entrance holes are covered with polyimide to provide a gas-tight enclosure.

The x-rays generated by the interaction of the laser with the Au hohlraum wall interact with the high-Z wall material via multiple absorption and re-emission to produce a near Planckian photon distribution with a temperature on the order of $200-\mathrm{eV}$ inside the hohlraum. The radiation heat wave (2) propagating through the hohlraum wall ablates the Au causing it to expand toward the center of the hohlraum. The pressure associated with the tamper retards the motion of the high $\mathrm{Z}$ plasma.

In this paper we discuss two experiments that study the effect of the tamper on the hohlraum. In the first experiment, we use a modified hohlraum to monitor the motion of the tamper-hohlraum interface as a function time. The hohlraum has $100 \%$ LEHs to permit an unobstructed view of the hohlraum wall. A gated imager is used to view the hohlraum along its axis by monitoring the $x$-ray emission from the high- $Z$ ions in three different energy bands (3). In these experiments, the laser beams are pointed in such a way that they illuminate the midplane circumference with ten-fold symmetry. This makes the illumination more "2-Dimensional" which is easier to model with the 2-D LASNEX (4) code and is a better approximation to the azimuthal illumination scheme intended for the National Ignition Facility (NIF). In the second experiment, we measure the effect of the tamper on the time dependent drive in a hohlraum. In these drive experiments we use a standard scale1 hohlraum (see below for details). The laser beams illuminate the hohlraum on either side of the midplane with five-fold symmetry, as depicted in figure 1 . A tenchannel, absolutely calibrated time-resolving spectrometer, Dante (5), is used to monitor the X-ray emission emanating from a small aperture in the hohlraum wall. This measurement provides the time history of the wall temperature inside the hohlraum, from which we can infer the drive temperature. In these experiments, we measure the Stimulated Brillouin Scattering (SBS) back into the lens of one of 
the ten Nova laser beams. Monitoring the scattered laser light helps us understand the overall hohlraum energetics.

\section{INTERFACE MOTION EXPERIMENTS}

To monitor the motion of the interface between the tamper and the ablated hohlraum wall material, we use a modified hohlraum that has $100 \%$ LEHs. Because of the large LEH diameter in these hohlraums, the windows are fabricated from $6500-\AA$ thick polyimide. Both the hohlraum diameter and length are 1600 $\mu \mathrm{m}$. The laser beams are pointed at the midplane of the hohlraum to provide tenfold symmetry in the azimuth. This provides a more uniform illumination of the wall, which is easier to model with a 2-dimensional code such as LASNEX. This is also a closer approximation to the anticipated illumination of a NIF hohlraum wall. The hohlraum is illuminated with a 2-ns long square pulse with a total energy of approximately $20 \mathrm{~kJ}$. The beam spot at the hohlraum wall is calculated to be about $675 \mu \mathrm{m}$ long and $460 \mu \mathrm{m}$ wide (in the azimuthal direction). The laser intensity at the wall is $-3 \times 10^{14} \mathrm{~W} / \mathrm{cm}^{2}$.

The primary diagnostic is the Soft X-ray Framing Camera (SXRFC) that views the hohlraum along its axis. This instrument is filtered to monitor emission around $450-\mathrm{eV}, 1200 \mathrm{eV}$ and $\mathrm{M}$-band emission (hv>2 keV). The SXRFC provides images at four different times in each of the three energy channels. Another framing camera views the hohlraum along its axis from the opposite side. This second camera has a fixed sweep length of $500 \mathrm{ps.} \mathrm{A} \mathrm{multiple} \mathrm{pinhole} \mathrm{array} \mathrm{on} \mathrm{the}$ front of the camera provides twelve images in the $\mathrm{M}$-band region during the 500-ps sweep. Figure 2 contains images from the SXRFC. We show here the $450-\mathrm{eV}$ channel at four different times during a pulse for two different hohlraum configura-

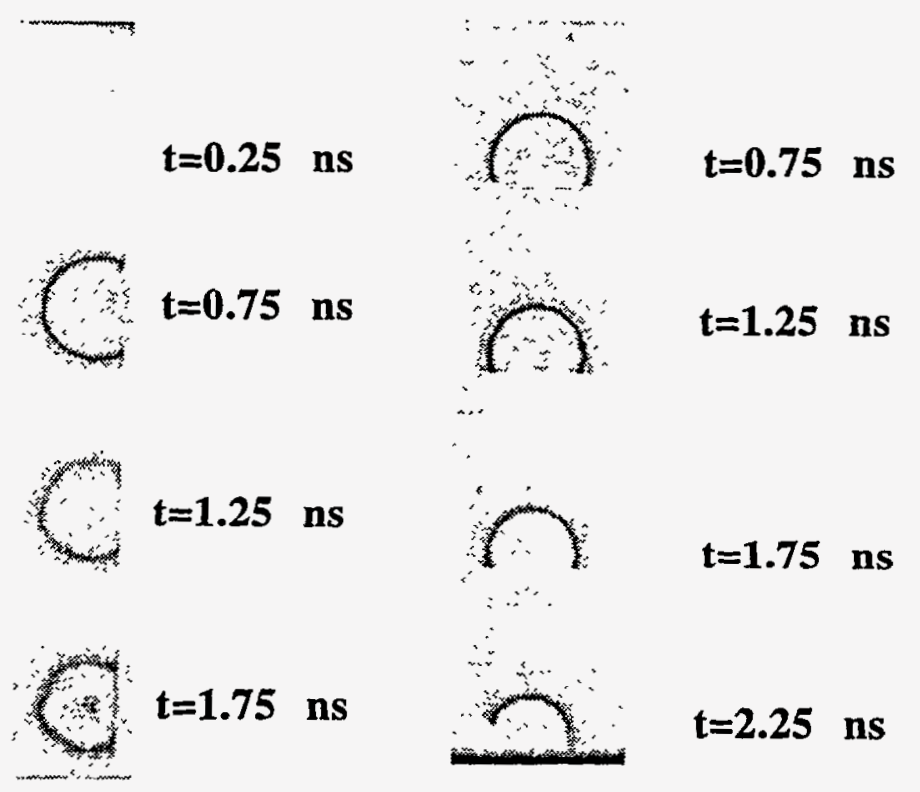

FIGURE 2. Axial images of an empty (left) and a neopentane-filled (right) hohlraum. These data correspond to the $450-\mathrm{eV}$ channel of the framing camera. 

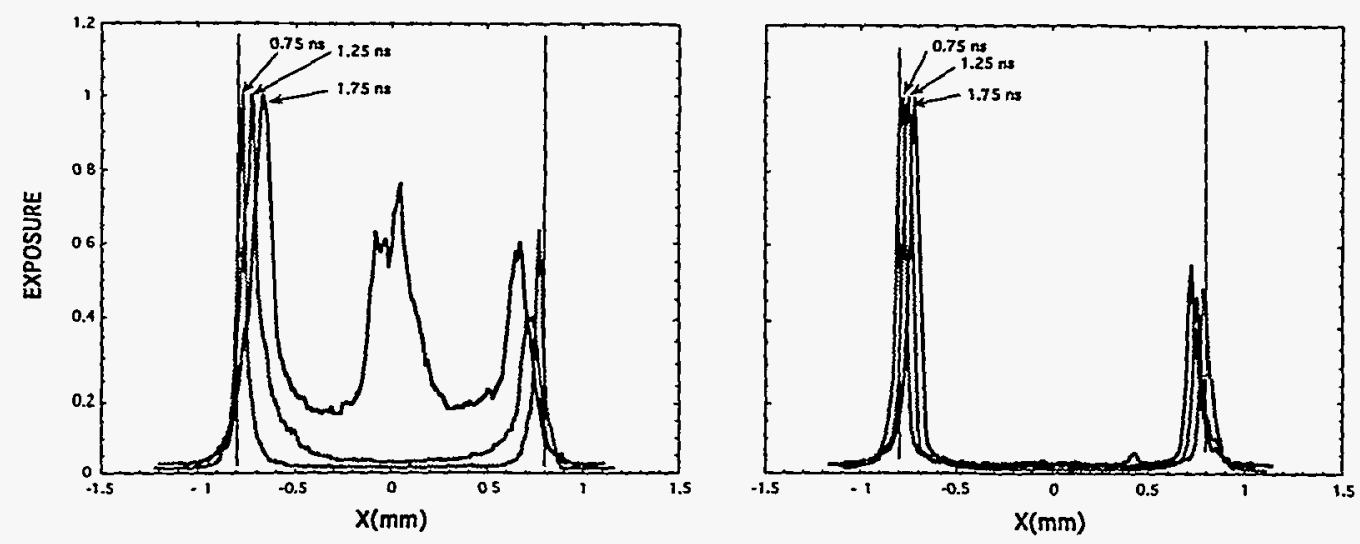

FIGURE 3. Lineouts of the images shown in figure 2. The exposure is in arbitrary units.

tions (windows on the LEHs but no gas fill and 1-atm $\mathrm{C}_{5} \mathrm{H}_{12}$ gas fill). Neopentane corresponds to $0.1 \mathrm{n}_{\mathrm{c}}$ when fully ionized. It is quite apparent that for the empty hohlraum the cavity fills with high-Z plasma and that toward the end of the pulse the plasma is stagnating on the axis. In contrast, the tamped hohlraums show the central region free of the emission corresponding to the high- $\mathrm{Z}$ material for the duration of the laser pulse. Lineouts of the axial images corresponding to the empty hohlraum and the $\mathrm{C}_{5} \mathrm{H}_{12}$ filled hohlraum are shown in figure 3 . The most apparent difference between the empty and gas filled hohlraums is the absence of low density gold filling the hohlraum. Not only does the $50 \%$ intensity point move in during the pulse, but the peak of the intensity moves in as well. This corresponds to the absorption region moving away from the original location of the wall. The data is in good agreement with LASNEX calculations, except in the M-band region where the code predicts the emission (and hence the location of the high- $Z$ material) to emanate from further inside the hohlraum (i. e. closer to the axis).

We determine the velocity of the interface by somewhat arbitrarily tracking the position of the $50 \%$-intensity point as a function of time. The velocity of the interface in the absence of the tamper is approximately $3 \times 10^{7} \mathrm{~cm} / \mathrm{s}$ while in the presence of the tamper generated from neopentane it is approximately $1 \times 10^{7} \mathrm{~cm} / \mathrm{s}$. However, we should point out that this method of quantifying the effect of the tamper totally neglects the fact that the low density, high-Z filling of the hohlraum is virtually eliminated by the tamper.

\section{HOHLRAUM TEMPERATURE MEASUREMENTS}

In order to determine the effect of the gas tamper on the hohlraum drive, We measured the temperature of a standard, scale-1 hohlraum without a tamper (or windows) and with a tamper generated from methane gas. These hohlraums are $1600-\mu \mathrm{m}$ in diameter and $2700-\mu \mathrm{m}$ long with $75 \%$ LEHs $(1200-\mu \mathrm{m}$ in diameter). The LEHs on the gas-filled hohlraums are covered with 3500 - $\AA$ thick polyimide. The laser pulse shape, shown in figure 6 , is designed for implosion experiments and incorporates a low-intensity foot followed by a peak in the drive. A ten 
channel, time resolving $x$-ray spectrometer views a portion of the interior hohlraum wall through a small aperture $(-400-\mu \mathrm{m}$ diameter) in the side of the hohlraum. The results of these measurements are shown if figure 5 for empty and methane-filled hohlraums. The effect of the tamper on the hohlraum temperature is two-fold: the peak of the drive is reduced about 20-eV and the "foot" of the drive is modified by the energy required to ionize and heat the gas. Preliminary investigations indicate that the gas species has little effect on the drive modification. That is propane and methane reduce the hohlraum temperature by about the same amount.

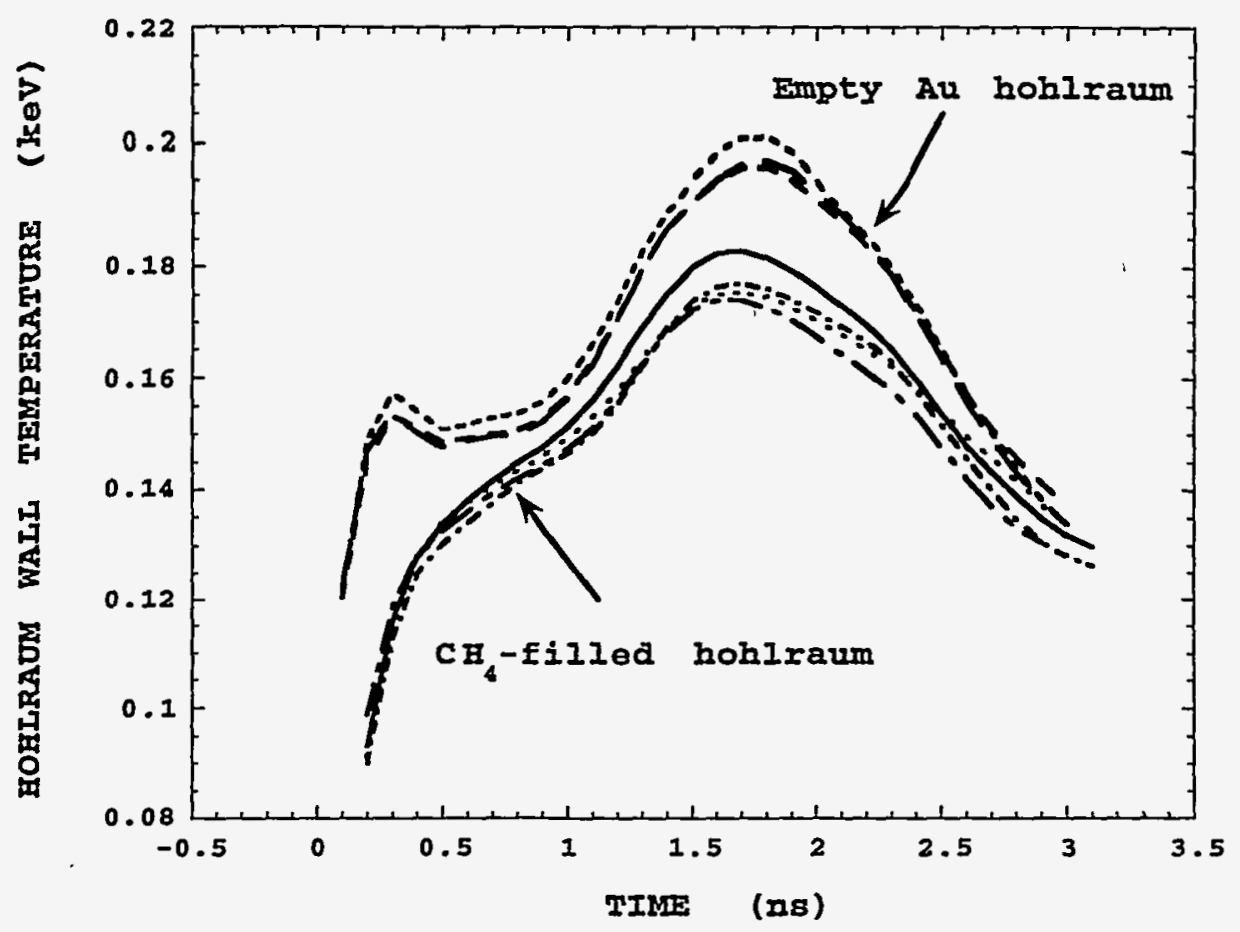

FIGURE 4. Hohlraum wall temperature as a function of time for empty and $\mathrm{CH}_{4}$-filled hohlraums.

In order to account for the temperature drop, we are measuring the reflected laser light from the hohlraum. We have made extensive measurements of the SBS and are beginning measurements of the SRS. Figure 6 shows the time dependent SBS that reflects back into a specific beam. We plot here the incident laser pulse (for a single beam), the SBS for a gas-filled hohlraum and for an empty hohlraum. Although the average SBS is nearly the same for the gas-filled hohlraum and the empty hohlraum, the temporal characteristics are markedly different. The empty hohlraum shows the SBS signal peaking in the latter part of the drive while the gasfilled hohlraum shows the peak of the SBS signal occurring during the front of the peak of the drive pulse. 


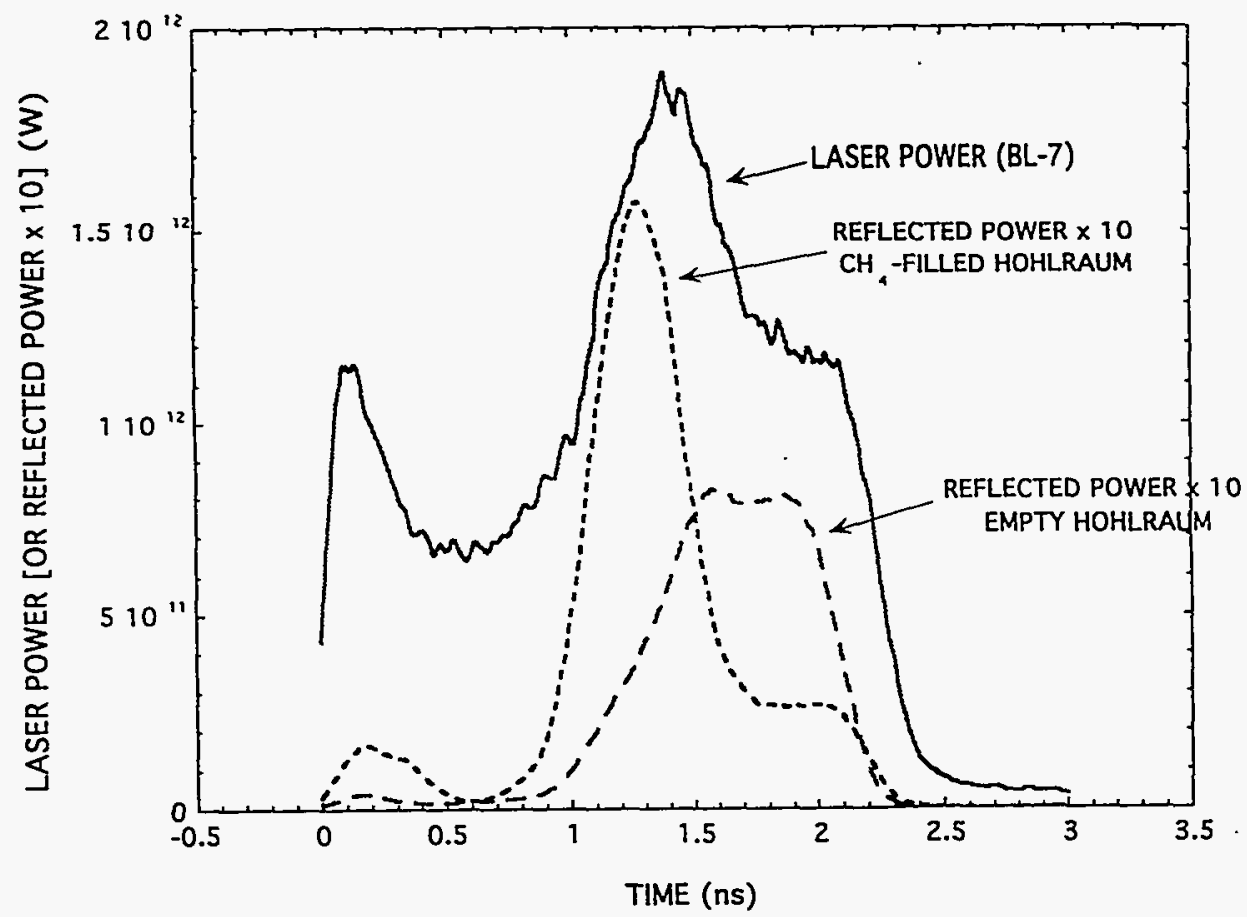

FIGURE 5. Laser pulse shape (power vs time) used in the hohlraum drive measurements. Also shown is the reflected SBS for an empty and a $\mathrm{CH}_{4}$-filled hohlraum.

\section{CONCLUSION}

We have measured the effect of the tamper in a gas-filled hohlraum. The ionized, low- $Z$ plasma is quite effective in preventing the high- $Z$ wall material from filling-in the hohlraum. We observe a temperature drop of about $10 \%$ when a tamper is introduced into the hohlraum. We are currently investigating the sources of this energy loss such as laser scattering via SBS and SRS. We also are investigating the properties of the low-Z tamper.

\section{REFERENCES}

1. Lindl, J. D., McCrory, R. L., and Campbell, E. M., Physics Today 45, 32 (1992)

2. Marshak, R. E., Physics of Fluids 1, 24 (1958)

3. Ze, F., Kauffman, R. L., Kilkenny, J. D., Wiedwald, J., Bell, P. M., Hanks. R., Stewart, J., Dean, D., Bower, J., and Wallace, R., Review of Scientific Instruments 63, 5124, (1992)

4. Zimmerman, G. B. and Kruer, W. L., Comments on Plasma Physics and Controlled Fusion 2, $51(1975)$

5. Kornbium, H. N., Kauffman, R. L., and Smith, J. A., Review of Scientific Instruments 57, 2179, (1986) 